\title{
Working out The Principles of Adaptive Management to Educational Processes in The E-Learning
}

\author{
R.N. Usmonov, V.S. Khamidov* \\ Computer Systems, Tashkent University Of Information Technology, Tashkent, 100000, Uzbekistan
}

\begin{abstract}
Suggesting the technology of intellectualization the process of adaptive management, based on situational analysis of educational process in the networking of single supporting system of decision making in E-learning.
\end{abstract}

Keywords Adaptive Management, Knowledge Base, Database, Term-Sets, Fasification, Defasification

\section{Introduction}

Strategy of decision making (DM) in the system of E-learning (EL) is defined by individual behavior of a trainee in the process of studying the appointed subject. At this integration the didactic potential of contemporary informational technology (CIT) in EL considered to be one of the leading aspects raising the efficiency of teaching process. Characteristics of educational process (EP) at this are the sequence producing extent of teaching materials, TM the content, quantity and types of control-checking works $(\mathrm{CChW})$, species and the methods of learning or adoption the TM. Working out and supporting of ordinary computerized education is defined by strategies creating the adequate level of knowledge of the trainee, educational Trass navigations that is not ought to be combined with informational combination of ordinary educational teaching materials. Hereof the barest necessity of optimization the process of training in EL which traditionally turn out to minimizing the time of training under the fixed level of learning or to the maximizing the content of learning under preserved time of teaching.

On this correlation below queries of intellectualizing processes of assistance accepted decision in EL on the foundation of integrating principles, theories of odd aggregate (TOA) are being considered. In informational relations the educational process in educational institutes is characterized by domination of subjective information's verbal character, which on the whole explained by relationship of pedagogic system to humanistic categories. Considerable part of information, concerning pedagogic systems, introduced in kind of verbal estimation of the majorities' data educational process, for example, such as the attendance by

\footnotetext{
* Corresponding author:

vkhamidov@gmail.com (V.S. Khamidov)

Published online at http://journal.sapub.org/am

Copyright (C) 2011 Scientific \& Academic Publishing. All Rights Reserved
}

students the classes, their progress in studies, the degree of learning by them the themes theoretic or practical hours and etc. The problem of receiving complex, objective assessment the quality of organization's educational process, requires utilization, in the process of making decision of all accessible information, including the quantitative information, (rating point of the students, the quality of missed hours and etc), and also quantitative (experience, the competence of the tutor, relation among different compound pedagogic system, introduced in the kind of linguistic points) characters. Directly and not indirectly, utilization in the process of modeling pedagogic processes the information of fuzzy character, presented in the form of verbal assessment the parameters of pedagogic system, considered to be an important moment in the achievement of modeling the basic goals - use of visual methods, certainty, objectivity.

\section{Main body}

On the one hand, formalization of pedagogic processes and phenomenon on the base principles of TOA enables to scrutinize methodologies, on the other hand - as a subject to solve specific pedagogic tasks, resolving on this gnosiological tasks, generally procedural and psychologically character. Gnosiological aspect of modeling methodology suppose applies in the process of cognition the pedagogic phenomenon; generally procedural aspect - estimate fuzzy connections and relations between component and date of pedagogic process, yet physiological aspect - describes variety side pieces of educational and pedagogic activity for the purpose of definition - psycho-pedagogical regularities.

Intellectual modeling activity of a trainee on the base of managing decision with the sequence of their realization in the real time is the basic proposing scheme of adaptive management. According to current layout, on the entry of systems given the information concerning the preparedness level of a trainee, organizational process training in EL, 
about the methods of a teaching and knowledge quality of a trainee. Trainees' preparation level (initial, current) is identified through the degree of learning by themselves TM. By this way, estimating the current advancement of a trainee while crossing from any to $\mathrm{k}+1$ learning $\mathrm{TM}$ cycle is a quite significant. The level organization process of teaching in E-learning depends on amenities of interface, introduced E-learning, the systems of organization and supervision of trainees' knowledge and etc. Readiness methods of assimilation TM depends on quality and quantity of proposing tests, teaching-methodological materials and classified as superficial, deep, absorbed.

The knowledge quality of a trainee - entire aggregate the steady attribute of knowledge, characterized by results cognitive activity of trainee, including current aspects:

Systematicness of knowledge, defined as integrating quality, being the result of conscious interaction, completeness, depth, certainty and generalization.

Efficacy of knowledge, visualizing in the ability of a trainee to use own knowledge in the solution of teaching and practical tasks, on top of that the tasks of vital character.

Solidity of knowledge, mastering on reflectivity and appearing in shaping the skills of self-analysis, self-assessment with consideration of others evaluation.

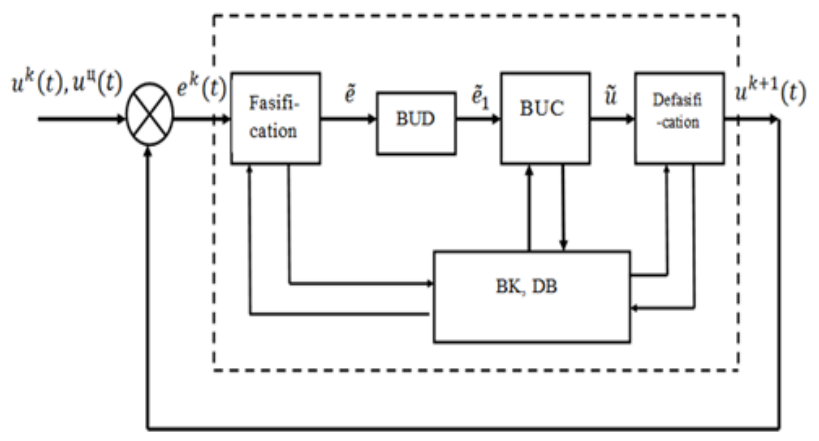

Figure 1. Schematic diagram of the fuzzy adaptive control of the EL. Here: BUD - block of fuzzy diagnosis the trainees' object research condition, BUC - block of fuzzy-logical conclusion: BK- base of knowledge, DB - Database.

Database. Let's move to exposition of constituting brought scheme of adaptive management

We shall bring in to the following designation:

$u^{k}(t)=\left\{x_{1}^{k}(t), x_{2}^{k}(t), x_{3}^{k}(t), y^{k}(t)\right\}-$ Targeting con-

dition the object's management: t- time;

$x_{1}^{k}(t)-<$ Preparedness level of a trainee $>$

$x_{2}^{k}(t)-<$ The level organization process teaching in E-learning $>$

$x_{3}^{k}(t)-<$ Mastering methods of teaching materials $>$

$y^{k}(t)-<$ The quality of teaching $>$

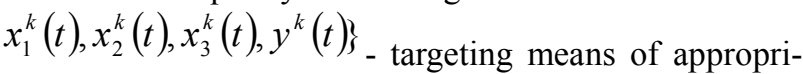

ate function, k-cycle teaching $\mathrm{K}-0,1,2 \ldots$;

$e^{k}(t)=u^{k}(t)-u^{k}(t)$-declining condition of MC from tar-

geting which in what follows defasifacate. If $\widetilde{e}$ accept as an fuzzy means $e^{k}(t)$, then $\widetilde{e}$ characterizes term;

$$
T(\widetilde{e})=\left\{T_{1}(\widetilde{e}), T_{2}(\widetilde{e}), T_{3}(\widetilde{e}), T_{4}(\widetilde{e}), T_{5}(\widetilde{e})\right\}
$$

where $T_{1}(\widetilde{e})-\langle V B R\rangle, \quad T_{2}(\widetilde{e})-\langle\dot{H} H\rangle, \quad T_{3}(\widetilde{e})-\langle H\rangle$, $T_{4}(\widetilde{e})-<B H>, T_{5}(\widetilde{e})-<3 B H>$. Herewith, VBR-vastly below rates; BR-below rates; R-rate;AR-above rates;VAR-vastly above rates.

These linguistical estimations of educational quality are defined by means of checking action, questioning on the base of conventional rates of evaluation which is accepted in the higher education system.

The base of given managerial system is a block of the ill-defined - logical conclusion which is oriented on realization of the following ill-defined model:

$$
Y=\widetilde{f}\left(x_{1}, x_{2}, x_{3}\right),
$$

where $x_{1}, x_{2}, x_{3}, y-$ parameters which is define the educational process, taken as linguistical changes (LC):

$x_{1}-<$ the level of a student's knowledge $>$;

$x_{2}-<$ the level of organization of the educational process ELS $>$;

$x_{3}-<$ the ways of the mastering of EM available in ELS>

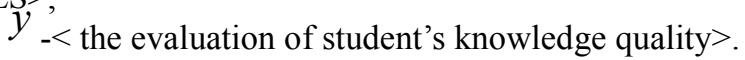

The Attributes ЛП $x_{1}, x_{2}, x_{3}, y$ - introduced by means of term-sets corresponding to universal sets.

Herewith,

$$
T\left(x_{i}\right)=\left\{T_{1}^{i}\left(x_{i}\right), T_{2}^{i}\left(x_{i}\right), T_{3}^{i}\left(x_{i}\right)\right\},
$$

As a matter of convenience we shall take the unitary indications, for all terms including LC at: L - low; A - average; $\mathrm{H}$ - high.

The Database in proposed system of adaptive management of the educational process of ELS includes the results to sampling, scaling, normalizations of universal sets ill-defined division of inputs and outputs (the amount of terms); the determinations of functional attributes (FA) terms of LS : $x_{1}, x_{2}, x_{3}, y-$ (the bell-shaped, triangular, trapezoidal etc.)

The knowledgebase presents itself system of the ill-defined rules, realizing ill-defined model of the type (1). Briefly we stop on linguistical estimations (the semantics) of terms LC $x_{1}, x_{2}, x_{3}, y \quad[6,8,9]$ :

$T_{1}^{1}\left(x_{1}\right)-\langle H\rangle \quad-<$ the student has a surface knowledge by studying subject, herewith the student tries to get any positive estimation with minimum expenses $>$;

$T_{2}^{1}\left(x_{1}\right)-<C>-<$ the student has firm knowledge, herewith he tries to get the knowledge, necessary for him for solving the standard problems in considered application domain>;

$T_{3}^{1}\left(x_{1}\right)-\langle B\rangle-<$ The student has enough high level of training, he tries to get the maximum of the knowledge required for practicing $>$;

$T_{1}^{2}\left(x_{2}\right)-<H>-<$ The process of the education in ELS is organized without considering the evaluation of the student's training level $>$;

$T_{2}^{2}\left(x_{2}\right)-\langle C\rangle \quad<$ In selecting EM of the educational process is taken into account the student's training level, but 
feedback is organized weakly $>$;

$T_{3}^{2}\left(x_{2}\right)-\langle B\rangle-<$ The educational process in ELS is organized by taking into account student's training level, the feedback is organized on high methodical level with provision for possible limits $>$;

$\left.T_{1}^{3}\left(x_{3}\right)-<H\right\rangle_{-}$- $<$Proposed way of the education is an inadequate to the student's training level $>$;

$T_{2}^{3}\left(x_{3}\right)-<C>-<$ Components forming subject of the intermediate checking knowledge is an inadequate to the student's getting knowledge degree during the training subject's sections $>$;

$\left.T_{3}^{3}\left(x_{3}\right)-<B\right\rangle-<$ The educational method is chosen by taking into account the student's estimation progresses during the training subject's sections $>$;

$\left.T_{1}^{4}(y)-<H\right\rangle \quad-<$ The student's knowledge does not match to the purposes of the education $>$;

$T_{2}^{4}(y)-<C>\quad-<$ The student has the necessary knowledge for solving the separate standard tasks, in ditto time the student has the gaps on using his knowledge for solving practical tasks $>$;

$T_{3}^{4}(y)-\langle B\rangle-<$ The student has enough strong knowledge he is able to use it for solving the concrete tasks $>$.

The realization of the ill-defined-logical model of the complex evaluation of the student's knowledge quality (1) is realized on the base of the designing the rule-oriented rules - a set of the controlling rules of the ill-defined logic, which is connecting inputs of the system with its output. Herewith is used taken above LC, and it's forming - the terms, as well as their ill-defined values. On the base incorporated by LC and its terms, the ill-defined model of the student's evaluation quality in ELS can be illustrated below:

If the student's training level $\left(\mathrm{x}_{1}\right)=(\mathrm{L}, \mathrm{A}, \mathrm{H})$,

And the level of organization of the educational process $\left(\mathrm{x}_{2}\right)=(\mathrm{L}, \mathrm{A}, \mathrm{H})$

And the methods of the mastering of $\operatorname{EM}\left(\mathrm{x}_{3}\right)=(\mathrm{L}, \mathrm{A}, \mathrm{H})$,

Then the quality of the knowledge $(\mathrm{Y})=(\mathrm{L}, \mathrm{A}, \mathrm{H})$,

The numeric evaluation of the student's knowledge quality $\left(y^{*}\right)$ for concrete values (numeric, linguistical) parameters in quantitative attitude is defined by the method of dephasification by the principle of the center of gravity [1,3-5].

The block of the diagnostics in educational process of adaptive management system is based on the determination of the taken decisions strategy on the base of the ill-defined analysis to situations, according to which whole information, incoming to input of the system $\left(e^{k}(t)\right)$ phasificates and is defined by an ensemble of standard situations, under which is understood the ensemble of the most typical situations, as well as the ensemble of purpose-oriented situations. The incoming to input of the block of the diagnostics phasificated information introduces in the manner of ill-defined current situation that is matched with purpose-oriented situation. As a result defined condition of the object of management, taken the controlling decisions, which are realized on the block of ill-defined-inference.

MDS process begins with AF determinations parameter situations:

$$
u^{k}(t)=\left\{\Delta x_{1}^{k}(t), \Delta x_{2}^{k}(t), \Delta x_{3}^{k}(t), \Delta y^{k}(t)\right\} .
$$

where:

$$
\begin{array}{r}
\Delta x_{i}^{k}(t)=x_{i}^{k}(t)-x_{i}^{u}(t) ; \\
\Delta y^{k}(t)=y^{k}(t)-y^{u}(t) ;
\end{array}
$$

On the base (4) are calculated current AF values [6]:

$$
\mu_{i}^{k}=\left\{\begin{array}{l}
\frac{a_{1}^{k}-\underline{a}_{i}}{\widetilde{a}_{i}-a_{i}}, \underline{a}_{i} \leq a_{i}^{k} \leq \widetilde{a}_{i} \\
\frac{\overline{a_{i}}-\underline{a}_{i}^{k}}{\overline{a_{i}-\widetilde{a}_{i}}}, \widetilde{a}_{i} \leq a_{i}^{k} \leq \overline{a_{i}}
\end{array}\right.
$$

Ill-defined estimates of $x_{1}, x_{2}, x_{3}, y$ indicators of object of the studies define under $\mu_{i}^{k}$ meaning. Herewith designs of the type are used:

$$
<\Delta \tilde{x}_{i}, X, \widetilde{C}_{(i)}>, \quad<\Delta \widetilde{y}, Y, \widetilde{C}_{(y)}>
$$

where

$$
\begin{gathered}
\widetilde{C}_{(i)}=\left\{<<\alpha_{i} / T_{1}^{i}>,<\beta_{i} / T_{2}^{i}>,<\gamma_{i} / T_{3}^{i}>\right\}, \\
\widetilde{C}_{(y)}=\left\{<<\alpha_{y} / T_{1}^{4}>,<\beta_{y} / T_{2}^{4}>,<\gamma_{y} / T_{3}^{4}>\right\}, \\
\underline{a}_{i}=\left\{\underline{\Delta x}, \quad \Delta \underline{y}_{i}\right\} ; \widetilde{a}_{i}=\{\Delta \widetilde{x} i, \Delta \widetilde{y}\}, \\
\bar{a}_{i}=\left\{\Delta \bar{x}_{i}, \Delta \bar{y}\right\}, \Delta \underline{x}_{i}<\Delta \widetilde{x}_{i}<\Delta \bar{x}_{i}, \Delta \underline{y}<\Delta \widetilde{y}<\Delta \bar{y}, \\
\underline{a}_{i}, \bar{a}_{i} \text { - lower and upper values of ill-defined num- }
\end{gathered}
$$
bers; $\Delta \widetilde{x}_{i}, \Delta \widetilde{y}$ on zero $\alpha$-level; $\widetilde{a}_{i}$ - average values of these numbers on single $\alpha$-level.

Adaptive management is realized on the base of the following ill-defined operators $[1,8]$ :

$<G, T_{G}, X>,<L, T_{L}, X>,\left\langle Z, T_{Z}, X>\right.$

where G, L, Z - LC, herewith , G- <intensify>, L- <reduce $>, \mathrm{Z}-<$ not to change $>X \in[-1,1]$

Forming terms - sets of $\mathrm{LC} \mathrm{G,} \mathrm{L} \mathrm{are}<$ little, vastly $>$;

The controlling decisions are designed on the base of the transformations of LC terms $\mathrm{G}, \mathrm{L}, \mathrm{Z}$ :

$$
\mathrm{R} 12: \quad T_{1}^{i} \rightarrow T_{2}^{i} \text {; R23: } T_{2}^{i} \rightarrow T_{3}^{i}, \quad i=\overline{1,3} .
$$

Herewith each operator Rij is put in correspondence to matrix, describing power of the influence of controlling decisions in term of values sign $\Delta \widetilde{x}_{i}, \Delta \widetilde{y}, i=\overline{1,3}$.

In particular for the term $R_{I_{1}}^{j} \in T_{G}-<$ little>, can be illustrated the matrix of the transition like this:

Let on sign $\Delta \widetilde{x}_{i} \in X$ have:

$$
\widetilde{C}_{(1)}=\{<0.9 / H>,<0.3 / C>,<0.2 / B>\}, i, j=\overline{1,3} \text {. }
$$

We shall use hereto condition operator R12 by means of $M_{I_{1}}^{j}$ 1 matrix then shall get:

$$
M_{I_{1}}^{j}=\begin{array}{l|lll|} 
& \text { H } & \text { C } & \text { B } \\
\hline \text { C } & 0.4 & 1 & 0.2 \\
0 & 0.5 & 1 \\
\text { B } & 0 & 0 & 1
\end{array}
$$


$\widetilde{C}_{(1)}=\{<0.4 / H\rangle,\langle 0.9 / C>,<0.3 / B>\}$ i.e. as a result of realization controlling operator, the student's knowledge level increased from category $0.9 / \mathrm{H}$ to $0.9 / C$ categories.

The efficient facility to realization of the complex approach in organizations, planning and management EP in ELS is a complex of programmes Fuzzy Logic Toolbox (FLT) functioning in ambience MatLab[3,5].

Modeling OP is in system FLT reasonable to divide into the following interconnected stages $[3,4,5]$ :

Determination input and output parameter to models;

Designing the database and knowledge for the development of the ill-defined model of the type Mamdani;

Undertaking computing experiment (CE) on the base of ill-defined built model;

Making the pedagogical decisions and recommendation on the base of the processing result $\mathrm{CE}$.

As a matter of convenience all input and output variable $(\mathrm{x} 1, \mathrm{~h} 2, \mathrm{~h} 3, \mathrm{y})$ are characterized alike terms-sets $\mathrm{T}=\{$ low, average, high $\}$, on unique universal sets marks [0,100].

The Ill-defined model of the evaluation of the student's knowledge quality is illustrated below:

$$
y=\widetilde{f}(x),
$$

In the process of the model realization (7) on FLT are accepted following indications:

$\mathrm{x} 1 \sim \mathrm{UPO} ; \mathrm{x} 2 \sim \mathrm{UOPO} ; \mathrm{x} 3 \sim \mathrm{SPOSOB} ; \mathrm{y} \sim \mathrm{CHOB}$.

The structure of model (1) in the FLT system with accepted indications is showed on fig. 2 .

Herewith, suitable interface and graphic possibilities of the system FLT is enable to plan and conduct VE on the base of designed ill-defined-logical model.

The series CE on the base FLT models is conducted, which is showed on Figure. 3.

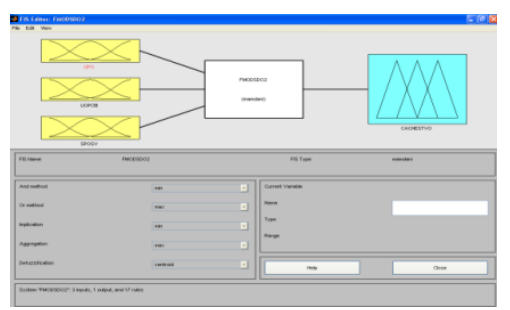

Figure.2. Structure to ill-defined model, the evaluation of the quality of $\mathrm{O}$ in FLT.

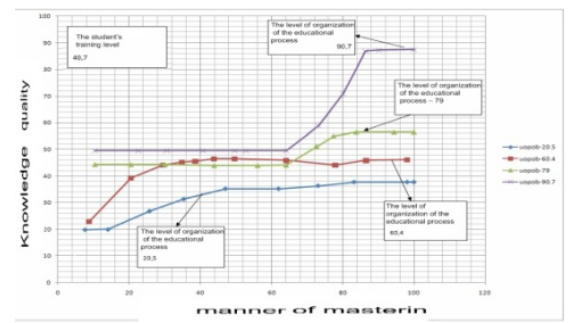

Figure.3. The results of $\mathrm{CE}$ on the base of interactivity model of OП in FLT.

The quality of the knowledge

1.The student's training level 40,7

2.The level of organization of the educational process 90,7

3. The level of organization of the educational process -79
4. The level of organization of the educational process 60,4

5. The level of organization of the educational process 20,5

The methods of the mastering of EM

The results of conducted by VE shows that at the student's training level, close to low $(\approx 40,7)$ more essential is a reinforcement of the work on introduction perspective technology education (Figure.3.)

\section{Conclusions}

Since one of defining elements of the ELS efficiency is an adequate determination of the student's knowledge level under k-ohm cycle of the education, that follows specifically to note the questions, connected with evaluation of the knowledge quality, including complex evaluation of such sides, as fullness, depth, operation indicator, flexibility, contrast, convolution indicator, systematic indicator, system regulation and realization of getting knowledge [8].

In conclusion we shall note that efficiency of the computer training systems is defined by designing methodical aspects evaluations of the student's knowledge level degree of learning subject, well-timed diagnostics discovery, the warning of backlog elements, as well as adaptive decision on a matter their removal.

\section{REFERENCES}

[1] Melikhov A., Bernstein J. C. Situation advising system with fuzzy logic. - Moscow: Nauka, 1990. - 272s

[2] VI Mikheev, Simulation and measurement theory in pedagogy.: Scientific-methodical manual for teachers, researchers, mathematicians, graduate students and researchers working on methods of ped. Research. - Moscow: Vyssh.shk., 1987. -2000 .; Ill

[3] Leonenkov A. Fuzzy modeling in MatLab and Fuzzy TECH. STDs.: BHV-Petersburg, 2003. -736 Sec.: Ill

[4] RN Usmanov On the question of integrating the theory of fuzzy sets in modeling pedagogical processes / Journal TUIT, № 1, 2009, p.113-118

[5] RN Usmanov On integration of fuzzy set-in approach to diagnose the state of complex systems / / Chemical Engineering. Control and management. - Tashkent, 2006, № 4, p.71-77

[6] S. Astanin, T. Kalashnikov. Development of individual student behavior in the system of distance education / / Advanced Information Technology and Intelligent Systems, № 1 (5), 2001, http://pitis.tsure.ru/

[7] A. Solovyov Designing computer systems for educational purposes: the manual. Samara: Samara State Aerospace University, 1995. 138

[8] The quality of students' knowledge and ways to improve it. Ed. Skatkin MN "Pedagogy", 1997, 208

[9] AN Alekseev Distance learning engineering degree: ITA "University Book", 2005, 333s 